\title{
A BOUNDARY VALUE PROBLEM IN THE THEORY OF PLASTIC WAVE PROPAGATION*
}

\author{
BY \\ E. H. LEE \\ Brown University
}

\begin{abstract}
In the analysis of boundary value problems in the theory of plasticity, the general situation arises that different partial differential equations are to be satisfied depending on whether the material is in the plastic or elastic state. The criterion determining the state at any material point depends on the dependent variables and their derivatives with respect to time. Thus the regions of application of the different differential equations must be determined from the boundary and initial conditions as the solution is developed.

The theory of the propagation of plastic waves in one dimension is a case in which the solution, including the determination of the unknown plastic-elastic boundaries, can be treated. An example is presented in this paper which illustrates the many types of boundary determination conditions which must be used. The method is based on the numerical integration along the characteristics of the hyperbolic equations arising, one linear and one quasi-linear. The development is possible since forward integration along characteristics enables the unknown boundaries to be determined independently of the subsequent solution. This situation is contrasted with other problems in the theory of plasticity. The complexity of the procedure indicates the difficulty to be anticipated with analytical treatment of such problems, and with the numerical treatment of problems involving more extensive plastic flow.
\end{abstract}

1. Introduction. The general mathematical theory of the propagation of plastic waves along rods was developed by Kármán, Bohnenblust and Hyers [1].** The solution of boundary value problems in this field requires the treatment of a quasi-linear wave equation in the regions where plastic flow is taking place, but the form of the equation changes if the stress falls at any section which has previously undergone plastic flow. Such a region is termed an unloaded or hysterises region, and its existence and development depends on the solution of the boundary value problem prior to the instant considered. This situation which requires the determination of the unknown boundaries between plastic and unloaded regions leads to a wide variety of boundary determination conditions. It is in this respect that the analysis differs from that of non-linear elastic waves in which the same differential equation would arise for both loading and unloading, so that the unknown boundary problem would not appear. The solution detailed below is presented since, in view of the wide variety of boundary determination conditions needed, it illustrates in a general way the types of problems to be faced in this field, emphasising the difficulty to be anticipated in the analytical attack on such problems.

The method of solution is based on the theory of characteristics of the hyperbolic equations arising, and is treated numerically. It was found that in order to maintain sufficient accuracy to determine the unknown boundaries between plastic and unloaded

*Received January 23, 1952.

**Numbers in square brackets refer to the bibliography at the end of the paper. 
regions it was necessary to compute the geometry of the slip line field using six significant figures. Graphical methods were found to be not sufficiently precise to determine consistent boundaries. Thus this is an example of the situation which may arise in the numerical treatment of boundary value problems, where more accuracy is needed to carry through the method than is warranted by the initial physical data: in this case the stress-strain relation of the material under consideration. It presumably means that in an experimental check minor variations in the material would involve appreciable changes in these boundaries. However in this case only minor changes in the final strain distribution would be expected to result.

Because of the accuracy required to complete the solution, it would be very lengthy to treat a problem involving more extensive plastic flow. Moreover, because of the variety of boundary conditions encountered, such problems do not lend themselves to routine computing.

2. Basic Theory. The equations governing the propagation of plastic waves were developed by Taylor [2] and Kármán [3] independently. Kármán's theory has recently been published with some experimental results (Kármán and Duwez [4]). The theory was later further developed principally by Bohnenblust in several O.S.R.D. reports. Here we shall consider the final form of the theory which applies for finite strain.

We are concerned with a material having an invariant relation between stress and strain under continued loading, independent of the speed of the test. Such a relation is shown by $O A B D$ in Fig. 1. In the elastic region $O A$ the relation is linear with gradient

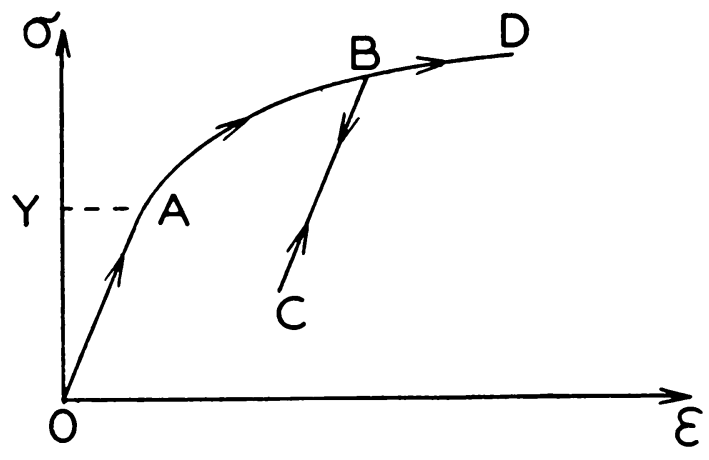

F IG. I

of magnitude Young's Modulus $E$. The arc $A B D$ represents plastic flow. The stress $\sigma$ is defined as the nominal stress, force per unit initial cross-sectional area, and $\epsilon$ the nominal strain, change in length per unit initial length. These nominal values are used, since in combination with the Lagrange type space coordinate $x$, large deformations can be analysed without introducing additional complexities into the equations. For convenience with the particular problem to be considered compressive stress and strain will be taken as positive. If when the point $B$ has been reached unloading takes place, elastic strain increments only occur, and the stress-strain point moves on the straight line $B C$ in Fig. 1 which is parallel to the original elastic line $O A$. If the stress later exceeds the value at $B$, the stress-strain point continues along the plastic flow curve $B D$. 
Longitudinal motion in the $x$ direction only is considered, and this puts a limitation on applications if the associated lateral motion becomes important. The equation of motion is

$$
\frac{\partial \sigma}{\partial x}=-\rho \frac{\partial^{2} u}{\partial t^{2}}
$$

where $\rho$ is the initial density, and $u$ the particle displacement from its initial position $x$ in the unstrained rod. If straining is taking place on the curve $O A B D, \sigma=\sigma_{p}(\epsilon)$, $\epsilon=-\partial u / \partial x ; \partial \sigma / \partial x$ can be replaced by $-\sigma_{\nu}^{\prime}(\epsilon) \partial^{2} u / \partial x^{2}$, and the quasi-linear wave equation is obtained:

$$
\frac{\partial^{2} u}{\partial t^{2}}=c^{2} \frac{\partial^{2} u}{\partial x^{2}}, \quad c^{2}=\frac{1}{\rho} \frac{d \sigma_{p}}{d \epsilon}=f(\epsilon)=f\left(-\frac{\partial u}{\partial x}\right) .
$$

The characteristics of this equation (see [1]) are given by $d x / d t=\mp c$, and along them we have the integral relation

$$
\frac{d x}{d t}=\mp c, \quad v \mp \varphi=\text { constant }
$$

where $v$ is written for the particle velocity $\partial u / \partial t$, and $\varphi(\epsilon)=\int_{0}^{\epsilon} c d \epsilon$.

When the material has been unloaded, the corresponding equations have been given by Bohnenblust [5]. If $B$ in Fig. 1 is given by $\left(\epsilon_{\max }, \sigma_{\max }\right)$, the unloading line $B C$ is

$$
\sigma=\sigma_{p}\left(\epsilon_{\max }\right)-E\left(\epsilon_{\max }-\epsilon\right) .
$$

The equation of motion becomes

$$
\frac{\partial^{2} u}{\partial t^{2}}=c_{0}^{2} \frac{\partial^{2} u}{\partial x^{2}}-\frac{d}{d x}\left[\frac{1}{\rho} \sigma_{p}\left(\epsilon_{\max }\right)-c_{0}^{2} \epsilon_{\max }\right],
$$

where $c_{0}^{2}=E / \rho$. The characteristics, and characteristic relations are

$$
\frac{d x}{d t}=\mp c_{0}, \quad \rho c_{0} v \mp \sigma=\text { constant. }
$$

At any specified time, the second term on the right hand side of (5) is a function of $x$ which is not prescribed initially, but which is determined from the solution at earlier times.

We have summarised the two types of equations to be considered. They can be applied directly to continuous stress fields as long as the stress strain relation $O A B D$ is concave downward. When this is not the case it has been shown by White and Griffis [6] and Lee [7] that shock waves are predicted, but we shall not be concerned with this situation in the present paper. However, in the elastic and unloading regions waves of stress and strain discontinuity can be propagated along the characteristics, and the Hugoniot relations of continuity and momentum change take on forms very similar to the characteristic relations. We shall be concerned with such finite linear waves.

We shall consider the solution in terms of the characteristic net in the $(x, t)$ plane and for convenience we replace the time coordinate by $\tau=c_{0} t / l$, and $x$ by $\xi=x / l$, where $l$ is a typical linear dimension. The linear characteristics are then given by $d \xi / d \tau=\mp 1$. The characteristic relations in the unloaded region $U$ are then

$$
\frac{d \xi}{d \tau}=\mp 1, \quad \rho c_{0} v \mp \sigma=\text { const. }
$$


and in the plastic region $P$

$$
\frac{d \xi}{d \tau}=\mp c^{\prime}, \quad \rho c_{0} v \mp \rho c_{0} \varphi=\text { const. },
$$

where $c^{\prime}=c / c_{0}$.

3. The impact problem considered. The problem we shall consider in detail, which involves an illustration of the many types of free boundary determination conditions which can arise in the analysis of plastic wave boundary value problems, is the motion resulting from the normal impact of a cylinder of length $l$, moving parallel to its axis with velocity $v_{0}$, against a rigid target at rest. For the convenience of having zero initial conditions we add a constant velocity $v_{0}$ to the whole system. The initial and boundary conditions are then

$$
\begin{aligned}
& v=0, \quad \sigma=0, \quad \tau=0, \quad 0<\xi \leq_{\mathbf{l}} 1, \\
& v=v_{0}, \quad \xi=0, \quad \tau>0, \\
& \sigma=0, \quad \xi=1, \quad \tau>0 .
\end{aligned}
$$

The initial development of the corresponding characteristics field is shown in Fig. 2. The discontinuity in velocity at 0 combined with the zero initial conditions determines a simple wave or lost solution with a fan of straight characteristics through 0 as dis-

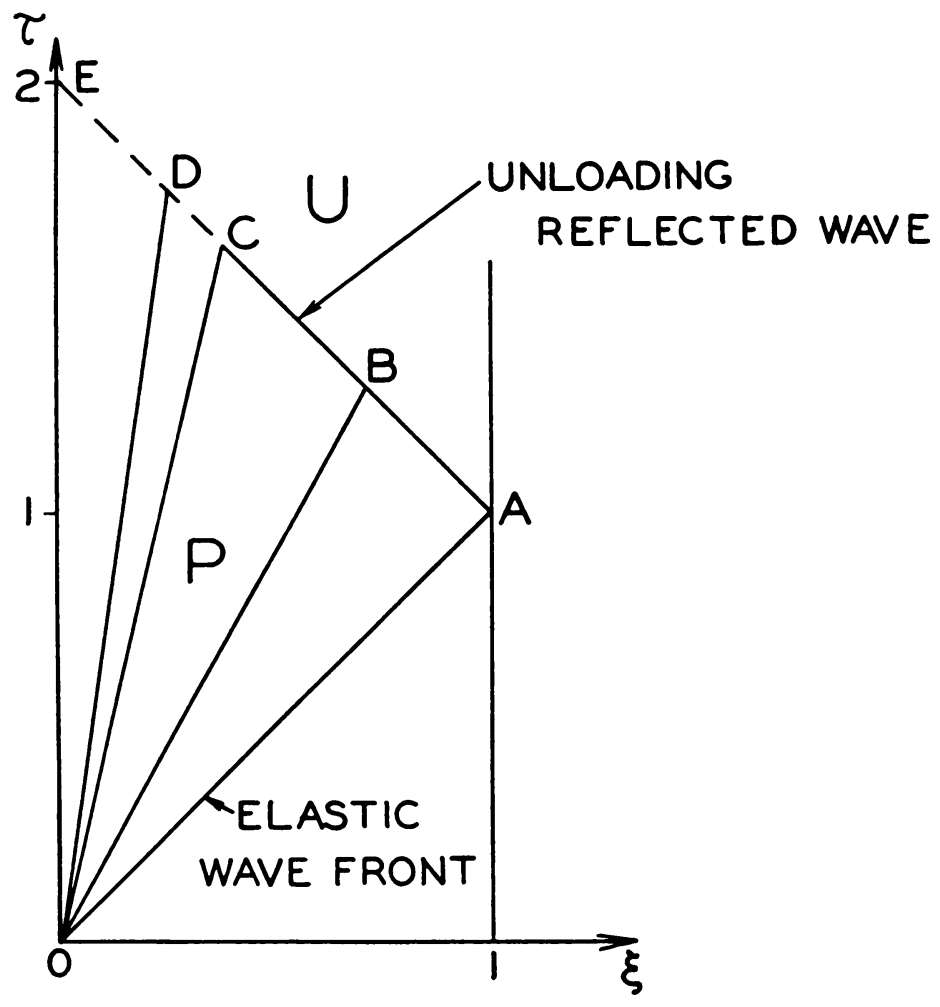

FIG. 2 
cussed by Kármán and Duwez [4]. Along each straight characteristic the velocity and stress remain constant. $O A$ is the elastic wave front across which the stress rises instantaneously from zero to the yield stress $Y$, corresponding to $A$ in Fig. $1 . O D$ is the characteristic corresponding to the velocity $v_{0}$. Throughout the triangle $O D E$ the material is moving with the constant velocity $v_{0}$ and is subjected to constant stress.

At the free end, $\xi=1$, the elastic loading wave is reflected at $A$ as an unloading wave of stress discontinuity $A B C$, which is propagated with the elastic wave speed $c_{0}$ in view of the unloading relation, and so has gradient -1 in Fig. 2. At $A$ the reflected wave is equal in magnitude and opposite in sign to the original elastic wave front and produces zero stress there, but as it advances into the plastic region its magnitude is reduced as discussed below. Let the suffixes $b$ and $a$ denote conditions before and after the unloading wave $A B C$ has traversed an element of the cylinder. Below $A C$ the material is in the plastic condition $P$, while above $A C$ unloading has taken place and the material is in the condition $U$. At any point along $A C \sigma_{b}, \epsilon_{b}$ and $v_{b}$ are known from the simple wave solution. The momentum relation for the unloading shock wave corresponding to the characteristic relation (6) is

$$
\sigma_{a}-\sigma_{b}=-\rho c_{0}\left(v_{a}-v_{b}\right) \text {. }
$$

At $A \rho c_{0} v_{b}=\sigma_{b}=Y$ for the elastic part of the initial wave solution. Since $\sigma_{a}=0$ at $A$, (8) determines $\rho c_{0} v_{a}=2 Y$ there. Considering the characteristic adjacent to $A C$, the constant in (6a) is given in terms of the known conditions at $A$, and we have

$$
\rho c_{0} v_{a}-\sigma_{a}=2 Y \text {. }
$$

Eliminating $v_{a}$ between (8) and (9) gives

$$
\sigma_{a}-\sigma_{b}=-\frac{2 Y-\left[\rho c_{0} v_{b}-\sigma_{b}\right]}{2} .
$$

Application of (3) for the initial wave solution emanating from 0 gives

$$
\rho c_{0} v_{b}=\rho c_{0} \int_{0}^{\epsilon b} c d \epsilon=\int_{0}^{\sigma_{b}} \frac{c_{0}}{c} d \sigma .
$$

since $d \sigma / d \epsilon=\rho c^{2}$. But $c=c_{0}$ in the elastic region $O A$ in Fig. 1, and $c<c_{0}$ when plastic flow occurs, and so it follows from (11) that the quantity $\left[\rho c_{0} v_{b}-\sigma_{b}\right]$ in (10) is zero at $A$ in Fig. 2 and increases from $A$ towards $C$. Thus (10) shows that the unloading shock wave is progressively absorbed as it penetrates the initial plastic wave front. If the impact velocity is below a certain critical value determined by (10), the unloading wave of stress discontinuity will continue through to the impact fact at $E$, but for higher impact velocities the unloading wave will be absorbed by the original plastic wave front. Thus, the stress discontinuity across the unloading wave may be reduced to zero at the point $C$, say.

Thus two types of problems arise in analysing the subsequent motion. The plastic region may spread from $C$ above $A E$, or the material may be unloaded throughout its length above $A C$. In the latter case it does not follow that plastic flow does not occur again. If at any section the solution of the equations for unloaded motion determines a stress equal to the previous maximum stress to which the section has been subjected, plastic flow can occur again there, and a new region in which the plastic wave equations must be solved is initiated. Such a problem is illustrated in this paper, and the dis- 


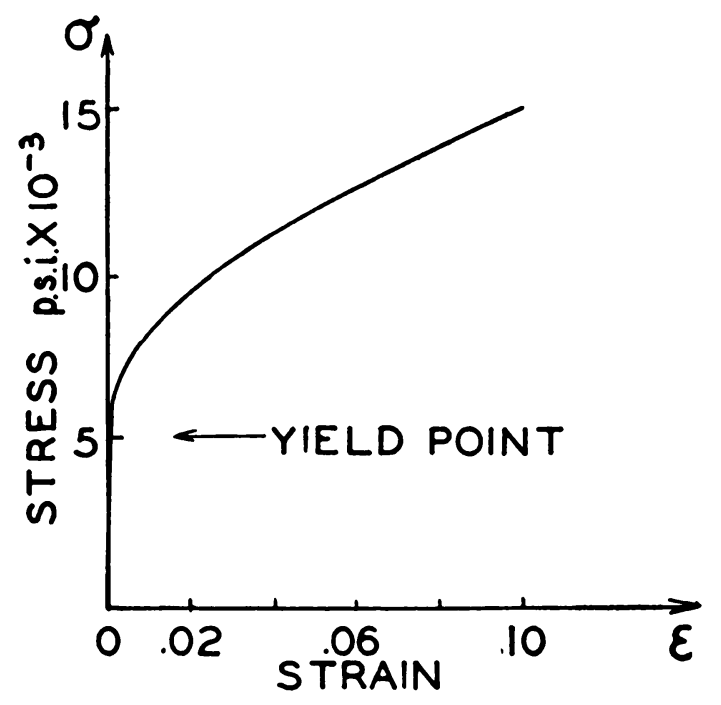

FIG. 3

cussion of the determination of the boundaries of such a region is given in the next section.

To illustrate the results in detail, the solution is presented for impact of a cylinder of aluminum having the stress-strain curve shown in Fig. 3. The corresponding $\varphi, \sigma$ relationship [see Eq. (3)] is shown in Fig. 4. For this material the unloading wave of stress discontinuity is propagated through to the impact fact for impact velocities $v_{0}<363 \mathrm{ins} / \mathrm{sec}$. For values greater than this the unloading wave is absorbed and the

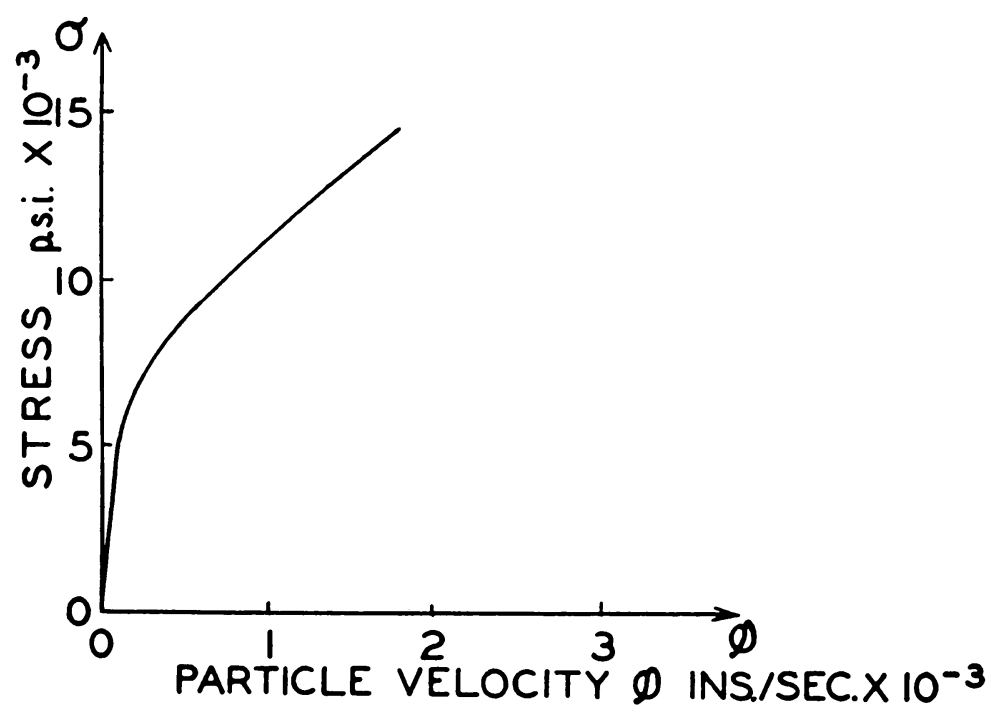


plastic region spreads out above it. The case considered in this paper, the characteristic field for which is illustrated in Fig. 5, corresponds to an impact velocity of 356 ins/sec so that the unloading wave just gets through without being absorbed, but for which plastic flow occurs again subsequently. The unloading wave is reduced from the yield point stress magnitude, $5 \times 10^{3}$ p.s.i., at $A$ in Fig. 5 to $0.131 \times 10^{3}$ p.s.i. along $B C$ where it remains constant.

4. Determination of the plastic-unloading boundary. For a material with a stress-

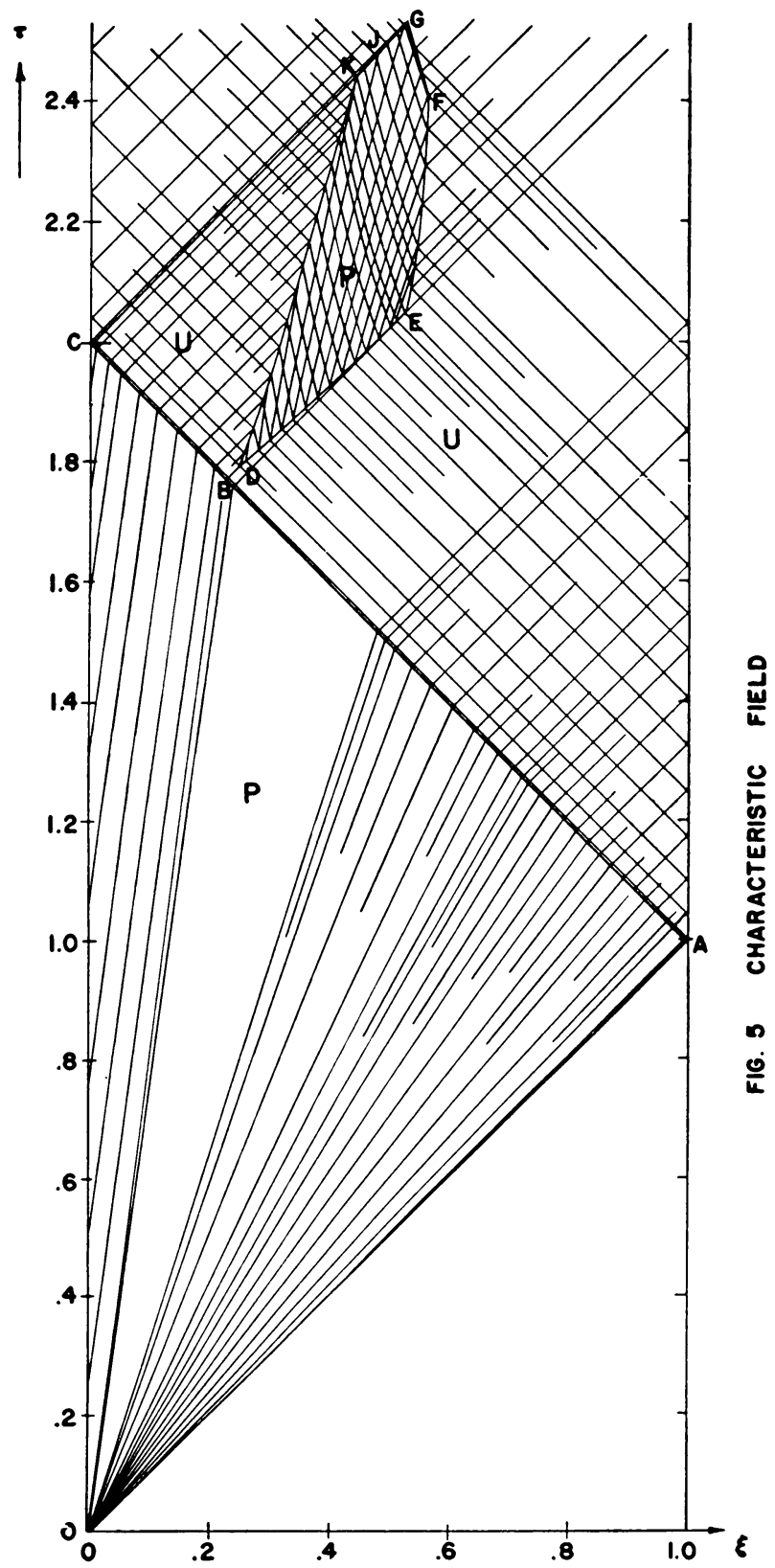


strain curve which is concave to the strain axis as considered here, shock waves of stress discontinuity are not initiated within the field of solution but must emanate from discontinuities in the boundary or initial conditions. Thus, if the unloading shock wave $A B C$ in Fig. 2 has been absorbed at $C$, the subsequent solution of the impact problem specified in (7) does not involve waves of stress discontinuity. However, discontinuities in stress and velocity derivatives may occur, and in fact are in particular associated with the continuation of the plastic-unloading boundary from $C$. Such discontinuities must therefore be considered in investigating the conditions which determine the boundary. A similar situation arises when a new plastic region is formed if the unloading wave $A C E$ in Fig. 2 traverses the entire cylinder. The conditions determining the plastic unloading boundary have been developed by Kármán, Bohnenblust and Hyers [1], but since this report may not be readily accessible, a brief statement of the theory is given in the Appendix with a somewhat simplified derivation.

In the previous section we considered the characteristic field shown in Fig. 5 up to the unloading shock wave $A C$. The solution beyond this can formally be continued by assuming the maintenance of the unloaded condition and making use of the characteristic relations (6). Before this extension can be considered satisfactory, it is necessary to check that the stress at any section does not exceed the previous maximum value reached just ahead of the unloading shock wave $A C$. This is, in fact, found to occur at $D$. The characteristic $O B$ determines the wave front of maximum stress, behind which in $O B C$ the stress is constant and equal to the maximum impact stress. In the unloaded region the influence of the wave front and the discontinuity of stress gradient across $O B$ is transmitted along the characteristic $B D$, leading to the initiation of the new plastic region at $D$. A similar condition for which according to the unloaded solution the stress reaches the new yield stress at each section determines the plastic-unloading boundary $D E$. This is a boundary of type (i) as defined in the Appendix, and since $d x / d t>c_{0}(d \xi / d \tau>1)$ no other conditions are required, and the two intersecting characteristics in $U$ and the known value of $\sigma_{\max }=\sigma_{b}$ on $A C$ determine its position. If these conditions are formally extended beyond $E$, it is found that $d x / d t<c_{0}$, and a new condition discussed in the Appendix must be used. It is, of course, evident that the old condition cannot be applied in this region since the characteristic $d \xi / d \tau=+1$ intersects the plastic region and so can no longer be used to determined the boundary. However, the formal continuation of the boundary $D E$ beyond $E$ is useful to obtain the termination point $E$ accurately. Since the boundary at $E$ is tangent to a characteristic $d \xi / d \tau=+1$, $\partial / \partial s\left(\rho c_{0} v+\sigma\right)$ is zero there, where $s$ is the arc length along the boundary, so that the position of $E$ can be determined from a plot of $\left(\rho c_{0} v+\sigma\right)$ extending on both sides of $E$. From the known conditions along the boundary $D E$ the solution in the plastic region is obtained by the method of characteristics for a Cauchy problem using finite differences and the characteristic relations (3).

A power series expansion of the variables about $E$ indicates that there is a discontinuity in the gradient of the boundary there. In fact $d x / d t$ becomes less than $c$, and the boundary $E F$ is determined by a characteristic in each of $P$ and $U$, and the known value of the yield stress at each section $\sigma_{\max }$. The determination of $E F$.permits the plastic field to be extended. At $F$ the boundary becomes vertical, so that a continuation would involve a type (ii) boundary as discussed in the Appendix and modified conditions must be satisfied. It is found that $d x / d t$ becomes $<c$, and the condition $d x / d t=$ $-1 / \rho(d \sigma / d v)$ must be used with a characteristic in each of $P$ and $U$ to determine $F G$. 
The upper part of the boundary $D K$ of the plastic region $P$ emanating from $D$ is determined by two characteristics in $P$ and one in $U . d x / d t>c$, so that with this type (ii) boundary no further conditions are to be satisfied. At $K$ this boundary intersects the remnant of the unloading wave reflected from the impact surface at $C$. The $P-U$ boundary then takes the form of an unloading wave of stress discontinuity with $d x / d t=$ $c_{0}$ along $K J$. The known solution in $P$, combined with the momentum relation and the characteristic value on the $U$ characteristic $K J$ determines the magnitude of the discontinuity across the unloading shock, and this is found to be completely absorbed at $J$. Thereafter, along $J G, d x / d t<c_{0}$, and two characteristics in $P$ and one in $U$ determine the segment of the boundary which closes the plastic region.

A check of the characteristic constants determined by the solution integrated to the point $G$, shows that the new yield stress at each section is not exceeded in the subsequent motion, so that the $U$ region covers the entire cylinder for all later terms. The cylinder will rebound in elastic vibration, with a permanent plastic strain distribution generated by the two plastic regions $P$.

5. Discussion of the solution. The permanent plastic strain distribution corresponding to the characteristic field of Fig. 5 is shown in Fig. 6. It is obtained from the maximum

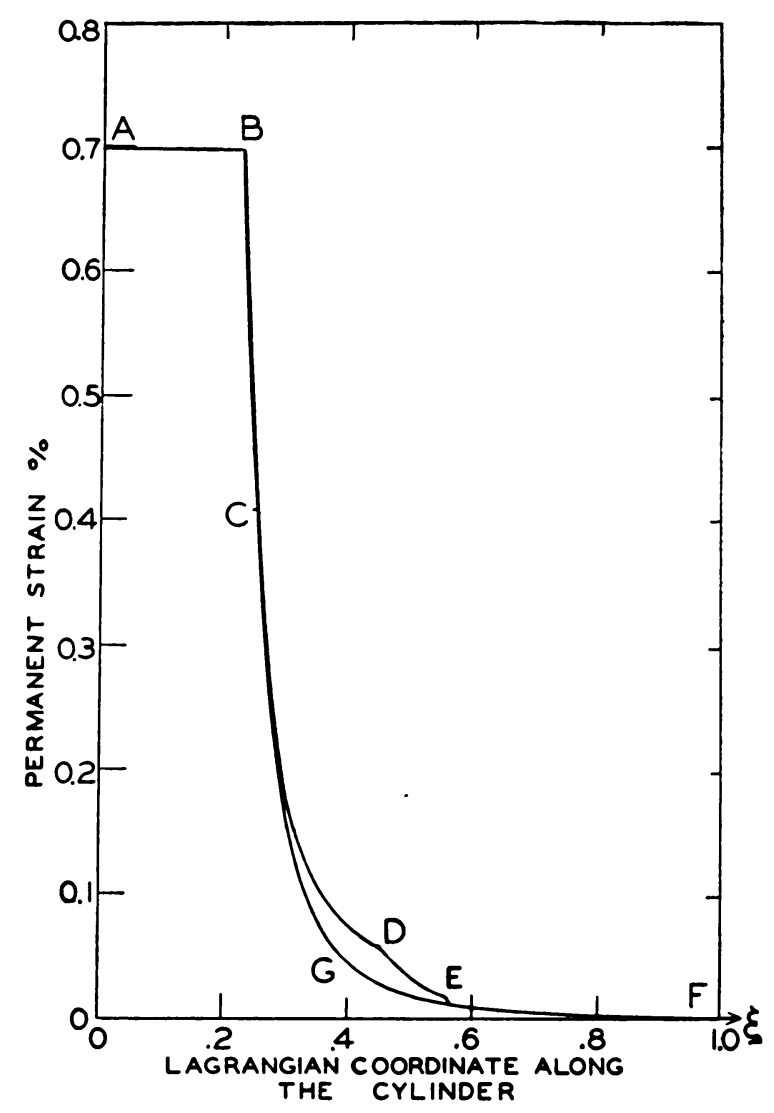

FIG. 6. THE PERMANENT STRAIN DISTRIBUTION 
strain reached at each section when plastic flow finally ceases there, by subtracting the elastic component of strain $\sigma_{\max } / E . A B C G E F$ is the plastic strain produced by the impact wave spreading from 0 in Fig. 5 before it is absorbed by the unloading elastic wave reflected from the free end. The subsequent flow due to the second plastic region produces the additional permanent strain CGED. This is seen to be small compared with that due to the primary plastic wave. The major part of the strain is seen to be concentrated at the impact end, which for higher velocities and large strains leads to the mushrooming effect of impact.

A study of this solution indicates the extensive detailed analysis required to determine the boundaries of the secondary plastic region even though it is quite limited in extent. It indicates the lengthy calculation to be expected for a case of higher velocity impact where detailed boundary considerations will be necessary for a much more extensive plastic region. The changing type of boundary, with the different conditions discussed in the Appendix, prevent a routine computational program and so increases the computing time. High numerical accuracy must be maintained in order to determine consistent boundary points, particularly on the segments where the condition $d x / d t=$ $-1 / \rho(d \sigma / d v)$ is used, since it involves, in its finite difference approximation, operating with the small difference between almost equal numbers. In the present case it was found necessary to work with six significant figures, and to compute all characteristic intersections in the $x-t$ plane, rather than making use of a graphical plot.

This problem illustrates the essential difficulty which arises in boundary value problems of the theory of plasticity. The different relations governing elastic deformation and plastic flow lead to different differential equations for material in these two states, and the domains through which each of the two states pertain must be determined from the prescribed boundary values on the outer boundary. This poses a much more difficult problem than the usual boundary value problem for a prescribed differential equation. The present problem of plastic waves in one dimension is a case that can be handled for general boundary conditions by the methods discussed above. The solution is made possible by the hyperbolic nature of the wave equations governing each type of behaviour, which permits forward integration along real characteristics, and the determination of the domains in which each equation applies at each stage as the solution proceeds with increasing time $t$. In other problems in the theory of plasticity such a direct procedure is not possible. For example, in plastic-rigid theory of quasi-static flow in plane strain, which has received considerable attention in recent years, different conditions are to be satisfied in the plastic and rigid regions. In the plastic region two hyperbolic pairs of first order partial differential equations arise which have the same pairs of characteristics, the slip lines. In general the prescribed boundary conditions are such that insufficient boundary information is available for forward integration of both pairs of equations, so that the solution at some point near the boundary may depend on the plastic-rigid boundaries to be determined within the domain. Thus, in general only very special problems can be treated, in which for example static determinacy permits separate integration of the stress pair of equations from the prescribed boundary conditions. From the point of view of considering the general types of boundary value problems in the theory of plasticity, it is useful to have one group of problems, waves in one dimension, for which general boundary value problems can be treated directly as illustrated in the present paper.

Acknowledgement. The solution discussed above was taken from an official report 
[7] written while the author was on the staff of the Armaments Research Department, Ministry of Supply, United Kingdom. Thanks are due to the Chief Scientific Officer, Ministry of Supply, for permission to publish this work.

Appendix. Determination of the P-U boundary.

As discussed in Section 4, we are concerned with a boundary across which derivatives of stress and velocity are discontinuous. For brevity we will use subscripts $x$ and $t$ to denote partial differentiation with respect to these variables, and superscripts $p$ and $u$ to denote values in the plastic and unloading regions.

Continuity implies that the differentials must be the same on the two sides of the boundary, therefore

$$
\begin{aligned}
& d \sigma=\sigma_{x}^{p} d x+\sigma_{t}^{p} d t=\sigma_{x}^{u} d x+\sigma_{t}^{u} d t, \\
& d v=v_{x}^{p} d x+v_{t}^{p} d t=v_{x}^{u} d x+v_{t}^{u} d t .
\end{aligned}
$$

Making use of the equations of motion and continuity $\sigma_{x}=-\rho v_{t}, \sigma_{t}=-\rho c^{2} v_{x}$, all derivatives but $\sigma_{t}$ can be eliminated from (a.1) giving:

$$
\frac{\sigma_{t}^{p}}{\sigma_{t}^{u}}=\frac{1-\frac{1}{c_{0}^{2}}\left(\frac{d x}{d t}\right)^{2}}{1-\frac{1}{c^{2}}\left(\frac{d x}{d t}\right)^{2}}=\alpha
$$

in which $\sigma_{t}^{p} \geq 0$ in order to maintain plastic flow. Two cases arise: (i) an element on the boundary is changing from $U$ to $P$, (ii) from $P$ to $U$. They are considered below side by side.

$$
\text { (i) } \quad \begin{aligned}
U & \rightarrow P, \\
\sigma_{t}^{u} & \geq 0 .
\end{aligned}
$$

From (a.2) $\alpha \geq 0$

$$
\left|\frac{d x}{d t}\right| \geq c_{0} \quad \text { or } \quad \leq c
$$

otherwise $\sigma_{t}^{p}=\sigma_{t}^{u}=0$. (ii) $\quad P \rightarrow U$,

$$
\sigma_{t}^{u} \leq 0 \text {. }
$$

From (a.2) $\alpha \leq 0$,

$$
c \leq\left|\frac{d x}{d t}\right| \leq c_{0}
$$

otherwise $\sigma_{t}^{p}=\sigma_{t}^{u}=0$.

When $\sigma_{t}^{p}=\sigma_{t}^{u}=0$, it follows from (a.1) that either $\sigma_{x}=0$ or

$$
\frac{1}{\rho} \frac{d \sigma}{d v}=-\frac{d x}{d t} .
$$

These conditions serve to determine the boundary as the solution is evaluated by characteristic integration.

\section{BIBLIOGRAPHY}

1. Th. von Kármán, H. F. Bohnenblust and D. H. Hyers, The propagation of plastic waves in tension specimens of finite length, NDRC Report No. A-103 (OSRD No. 946), 1943.

2. G. I. Taylor, Propagation of earth waves from an explosion, British Official Report, R.C. 70, 1940, The plastic wave in a wire extended by an impact load, R.C. 329, 1942.

3. Th. von Kármán, On the propagation of plastic deformation in solids, NDRC Rebort No. A-29, OSRD No. 365. 1942. 
4. Th. von Kármán and Pol Duwez, The propagation of plastic deformation in solids, J. Appl. Phys. 21, 987-994 (1950).

5. H. F. Bohnenblust, Comments on White and Griffis' theory of the permanent strain in a uniform bar due to longitudinal impact, NDRC Memo. A-47M, OSRD No. 781, 1942.

6. M. P. White and L. Griffis, Wave propagation in a uniform bar whose stress-strain curve is concave upward, NDRC Report No. 152, OSRD 1302, 1943, J. Appl. Mech. 70, 256 (1948).

7. E. H. Lee, Plastic waves in compression, British Official Report A.P.P., Co-ord. Sub-Committee No. 57, 1943. 